\section{Palobra}

acceso $\partial$ abierto

Cómo citar: Correa, R., Vidal, L., Marmolejo, E., Sánchez, C. (2020). Procesos de inclusión en la educación superior en Colombia, México y Chile. Palobra, 20(1), 96-112. https://doi.org/10.32997/2346-2884-vol.20num.1-2020-3227

Recibido: 17 de diciembre de 2019

Aprobado: 31 de enero de 2020

Editora: Rosario Blanco Bello. Universidad de Cartagena-Colombia.

Tipología IBN Publindex: Artículo de Revisión.

\title{
Procesos de inclusión en la educación superior en Colombia, México y Chile ${ }^{1}$
}

\author{
Inclusion processes in higher education in Colombia, Mexico \\ and Chile
}

\author{
Rodney Correa Jaramillo \\ Universidad Católica Luis Amigó-Colombia, rodney.correaja@amigo.edu.co \\ Laura Andrea Vidal Yepes \\ Universidad Católica Luis Amigó-Colombia, lavy-14@hotmail.com \\ Efraín Arnoldo Marmolejo Sarmiento \\ Universidad Católica Luis Amigó-Colombia, efrain.marmolejosa@ amigo.edu.co \\ Claudia Nayibe Sánchez Moncayo \\ Universidad Católica Luis Amigó-Colombia, claudia.sanchezmo@amigo.edu.co
}

\section{RESUMEN}

La educación inclusiva viene naturalizando su discurso al permear la esfera social e institucional, convirtiéndose en la razón por la cual las acciones educativas sean resignificadas en el marco de la flexibilidad. Esto genera transformaciones sociales que son cada vez más vertiginosas, permitiendo así que el contexto político extienda su plataforma al incorporar la cultura de la diversidad, lo que visibiliza la población vulnerable. Esta pluralidad en Latinoamérica ha generado reformas educativas, desde el diseño universal del aprendizaje hasta el desarrollo de los proyectos educativos institucionales (PEI) ${ }^{2}$. A esta importante tarea se sumaron países como Chile, México y Colombia, quienes han implementado dentro de su marco político el concepto de educación inclusiva, como un primer paso para impulsar el avance y la mejora de todas las personas sin ningún tipo de discriminación. La exhaustiva revisión bibliográfica dejó ver cómo la inclusión en la educación superior, en Chile, México y Colombia, se fundamenta en la normatividad legal, que exige a las instituciones de educación superior contar con herramientas que permitan la enseñanza a toda la población.

Palabras clave: Educación superior; educación inclusiva; políticas públicas; población vulnerable; currículo.

Copyright: $\odot$ 2020. Correa, R., Vidal, L., Marmolejo, E., Sánchez, C. Este es un artículo de acceso abierto, distribuido bajo los términos de la licencia https://creativecommons.org/licenses/by-nc-sa/4.0/ la cual permite el uso sin restricciones, distribución y reproducción en cualquier medio, siempre y cuando que el original, el autor y la fuente sean acreditados.

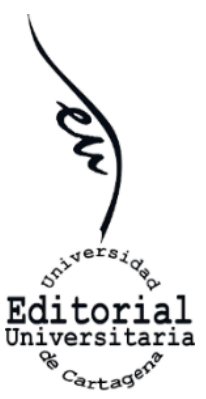

\begin{abstract}
Inclusive education has been naturalizing its discourse by permeating the social and institutional sphere, becoming the reason why educational actions are resignified within the framework of flexibility. This generates social transformations that are increasingly dizzying, thus allowing the political context to extend its platform by incorporating the culture of diversity, which makes the vulnerable population visible.

\footnotetext{
${ }^{1}$ Este artículo es derivado de la investigación titulada, "Procesos de inclusión en la educación superior en Colombia, México y Chile”, la cual fue financiada con recursos propios.

${ }^{2}$ Proyecto Educativo Institucional (PEI) es el documento que demarca el horizonte en todas las instituciones educativas
} públicas y privadas en Colombia.
\end{abstract}


This plurality in Latin America has generated education reforms, from the universal design of learning to the development of institutional education projects (PEI). This important task has been joined by countries such as Chile, Mexico and Colombia, which have implemented within their political framework the concept of inclusive education as a first step towards promoting the advancement and improvement of all people without any kind of discrimination. The exhaustive bibliographic review showed how inclusion in higher education in Chile, Mexico and Colombia is based on legal regulations, which require higher education institutions to have tools that allow them to teach the entire population.

Keywords: Transformation; inclusion; public policies; vulnerable population; curriculum.

\section{INTRODUCCIÓN}

Las necesidades educativas derivan de las transformaciones sociales que ocurren dentro de un contexto determinado, lo cual condiciona el planteamiento de nuevas estrategias para dar soluciones oportunas y eficaces, que no desentonen con las características de la realidad. La educación como proceso transformador se viene adaptando a estos cambios sociales, generando un continuo desarrollo. Una de las necesidades más apremiantes en los últimos tiempos es la superación de los paradigmas que sustentan las teorías del aprendizaje, con su filosofía escolástica como plataforma principal de la educación, la cual, por años, se ha mantenido inmutable, evadiendo toda transformación. Esta hegemonía viene perdiendo fuerza ante el incremento discursivo de los procesos de inclusión y de flexibilidad curricular; la inclusión es definida como el conjunto de "acciones e intervenciones que promueven el acceso a derechos y espacios en igualdad de condición" (Ceballos y Acosta, 2015, p. 112), por tanto, permite la participación de toda la sociedad sobre las transformaciones del país.

Las ideas expuestas en el párrafo anterior son complementadas por Carrizosa (s.f.), quien considera que la inclusión debe iniciar con el respeto por la participación de todos los pensamientos. En el ámbito educativo, la Organización de Naciones Unidas -ONU- (2018), expone que la inclusión "desde el punto de vista de los derechos de las personas con discapacidad", significa la igualdad de oportunidades para alcanzar la participación, garantizando el acceso igualitario a los procesos educativos. Este pensamiento filantrópico contradice la realidad social planteada por Lissi et al. (2009), cuando afirman que en Chile el 6,6\% de los jóvenes con algún tipo de discapacidad accede a la universidad. No obstante, se topan con barreras que involucran aspectos como la infraestructura, la comunicación o el aprendizaje mismo, lo cual entorpece y dificulta su estabilidad y permanencia.

La diversidad cultural que caracteriza la población colombiana ha venido generando transformaciones educativas que, aunque son paulatinas, evidencian el interés de las comunidades académicas en realizar 
contribuciones importantes para promover la inclusión, como garante de derechos de todos los ciudadanos.

La Consejería Presidencial para el Desarrollo Institucional (1996) explica que, desde los años 90, la misión de los sabios se enfoca en la búsqueda de las mentes más brillantes del país, en diferentes áreas del conocimiento, para que intervengan en el plan de desarrollo educativo y profundicen en los aspectos de la ciencia, la tecnología y la innovación, en aras de dar respuesta a los desafíos sociales, y así lograr el crecimiento del país. En la actualidad, Colombia se encuentra en una importante transición educativa que tiene como pilar estructural el acceso a la educación para toda la población.

El concepto de inclusión en Colombia, desde el punto de vista legislativo, data del año 1994 con la promulgación de la Ley 115 -Ley General de Educación-, y se fortaleció en 1996 con la expedición de la Ley 324, referida a la normatividad en favor de la población sorda, y en 1997, cuando fue expedida la Ley 361 que exhortó a la sociedad, incluyendo a las instituciones educativas, a crear mecanismos de integración social para las personas con limitaciones. Estas reglamentaciones explicitaron las formas en que el Estado garantizaría, por medio de recursos pedagógicos, apoyo a la población con discapacidad física o psicológica, mediante acciones pedagógicas y terapéuticas para el acceso al proceso educativo, así como los mecanismos de culturización para garantizar la formación integral.

La metodología aplicada se realizó mediante un diagrama con los criterios de selección de las referencias rastreadas, especificando las razones por las cuales fueron seleccionadas. Finalmente, se evidencian los aportes más significativos que dieron lugar a la consolidación de la temática central del presente artículo.

\section{Impacto de las transformaciones sociales de la universidad latinoamericana en el discurso sobre la inclusión en la realidad colombiana}

La inclusión en el ámbito educativo fue instaurada, de manera formal, por la Organización de las Naciones Unidas para la Educación, la Ciencia y la Cultura-UNESCO- en 1960, bajo el tratado internacional de la lucha contra las discriminaciones en la esfera de la enseñanza. Promulgó la necesidad de plantear la educación como un derecho inalienable accesible para toda población. Este discurso ha impactado todas las esferas educativas, siendo acogido por los países del mundo como guía para el mejoramiento de la vida humana, por medio de la ciencia y la culturización. Estos avances en la educación se pueden visualizar en los Objetivos de Desarrollo Sostenible (2015-2030), planteados por el Programa de las Naciones Unidas para el Desarrollo -PNUD-. Este compendio legal es un llamado universal para poner fin a la pobreza, proteger el planeta y garantizar que todas las personas 
gocen de paz y prosperidad para 2030. Específicamente, en términos educativos, para esta fecha, los estudiantes deben tener conciencia de la importancia de la educación de calidad para todos y deben concebirla como un bien público, un bien común mundial y un derecho humano fundamental.

El impacto de las políticas universitarias en materia de derechos ha promovido adaptaciones curriculares que fortalezcan el diseño universal del aprendizaje, para garantizar el acceso al conocimiento de todos los estudiantes, de tal manera que se dé respuesta a las nuevas demandas sociales que agitan los países latinoamericanos. Al respecto, Brunner (1990), Mollis (2006) y Forero, González y Gómez (2007) manifiestan que las trasformaciones educativas en educación superior, en el contexto latinoamericano, han pasado por una serie de evoluciones que van desde los planteamientos escolásticos sometidos al clero, hasta nuevos ideales que, a través de la adopción de modelos alternativos de desarrollo, generan tránsitos hacia nuevas identidades.

Sin embargo, es importante reconocer que las universidades latinoamericanas no han escapado al enfoque mercantilista que ha invadido y trastocado su función primordial formativa, para dar paso a una finalidad demarcada por la productividad. Esta intención ha provocado el declive del saber, en términos filosóficos, técnicos y científicos, para otorgar privilegios al saber financiero y al cultivo de las élites, como fuente de promoción de la desigualdad y la inequidad en el acceso a las oportunidades laborales y educativas.

Estas ideas son retomadas por Gómez (2000), quien expone que el Estado colombiano, con su pensamiento neoliberal, está siendo debilitado por el clientelismo, y es incapaz de proponer escenarios deseables, en relación con las nuevas demandas de la educación universitaria. Estas vicisitudes orillan a las universidades en función del mercado y la oferta. Tales dinámicas sociales, sumadas a las nuevas realidades del país, han coartado el acceso a la educación superior de importantes grupos poblacionales, para quienes pareciera que los garantes de derecho no tienen funcionalidad.

En este sentido, la necesidad de reformas educativas en Colombia ha respondido, según Bocanegra (2011) y Flórez (2016), al rezago del país frente a las necesidades de desarrollo social e intelectual. Los modelos planteados para suplir las debilidades, referidas a la calidad educativa, han generado cambios que resultan insuficientes en términos de calidad, cobertura y oportunidad. Los mayores avances se han visto en la implementación de nuevas políticas educativas como derecho fundamental; dentro de la legislación expedida, se subraya la inclusión de toda la población en los contextos educativos. Este tránsito de la vulnerabilidad al reconocimiento de derechos, es examinado por autores como Arizabaleta y Ochoa (2016), quienes ubican la educación inclusiva en el marco de una gestión transformadora, que se adapta a las diferentes limitaciones del ser humano. 


\section{Marco político del concepto de inclusión educativa en Colombia, México y Chile: relación entre sus discursos}

La inclusión como discurso en el ámbito educativo en Colombia ha pasado por una serie de transformaciones, en respuesta a las realidades que vive el país. El artículo 67 de la Constitución Política de 1991, así como la Ley 30 de 1992, manifiestan que la educación es un derecho y un servicio en pro de la sociedad, por tanto, posibilita el desarrollo integral del ser humano. Muchos de los discursos sobre la inclusión y la educación inclusiva superior, que se encuentran implícitos en la Constitución Política, fueron retomados de países latinoamericanos que han vivido carencias similares al contexto colombiano. Con respecto a México, este inició un proceso de educación inclusiva con una modalidad de integración en 1993; García et al. (2003), en su investigación, dio a conocer que los avances en la educación especial eran muy pocos, lo cual dio inicio al Proyecto Nacional de Integración Educativa (PNIE), este proyecto tuvo gran acogida entre los maestros, por lo que, en el 2002, nació el Programa Nacional de Fortalecimiento de la Educación Especial y de la Integración Educativa (PNFEEIE).

Por su parte, en cuanto a Chile, La Ley General de Educación (LGE), número 20.370, promulgada el 17 de agosto de 2009, menciona en su artículo $\mathrm{N}^{\circ} 1$ :

Sobre derechos y deberes de los integrantes de la comunidad educativa, fijando los requisitos mínimos que deberán exigir cada uno de los niveles de educación parvularia, básica y media, velando por el cumplimiento, requisitos, y el proceso del reconocimiento oficial de los establecimientos es instituciones educacionales, con el objetivo que apunta a la equidad y calidad del servicio. (Párr. 1).

Así las cosas, la legislación chilena, de acuerdo al Ministerio de Educación de Chile en la ley mencionada, hace alusión al tipo de Educación Especial, donde se determina lo siguiente:

La Educación Especial o Diferencial es la modalidad del sistema educativo que desarrolla su acción de manera transversal en los distintos niveles, tanto en los establecimientos de educación regular como especial, proveyendo un conjunto de servicios, recursos humanos, técnicos, conocimientos especializados y ayudas para atender las necesidades educativas especiales que puedan presentar algunos alumnos de manera temporal o permanente a lo largo de su escolaridad, como consecuencia de un déficit o una dificultad específica de aprendizaje. (Art. 23, párr. 1).

En esta perspectiva legislativa, Queupil y Durán (2018), manifiestan que la educación superior chilena se moderniza y reforma con las políticas correspondientes, teniendo presente el principio de la inclusión, por creciente demanda a la educación del país. 
Las ideas precedentes las retoma el Ministerio de Educación chileno al manifestar que la educación superior en el país impulsa el avance de todas las personas sin discriminaciones, para que puedan desarrollar talentos; por tal pensamiento, no es extraño que Chile sea referente en educación superior en Latinoamérica. Esto lo explican Rivas y Sánchez (2016) después de realizar un rastreo en siete países latinoamericanos y analizar los resultados en las pruebas PISA del 2000 al 2015; no en vano se evidencia que Chile es el país más destacado en América Latina, en el ámbito educativo, con los niveles más bajos de repetición y los más altos en titulaciones.

Además, uno de los elementos que posiciona a Chile como un país modelo, en el marco educativo, es el financiamiento de la educación; Marcel y Tokman (2005) enuncian que la educación chilena es patrocinada por los entes públicos, y aunque el sector privado también es actor en el financiamiento educativo, el tesoro público sigue siendo el principal ingreso de la educación. Para contrastar esta idea, Villalobos y Quaresma (2015) manifiestan que el método de financiamiento para la educación en Chile es ineficiente, porque "genera una dimisión del rol del Estado, la centralidad de lo privado, la implementación de subsidios a la demanda y la generación de fuertes políticas de rendición de cuentas" (pág. 1), lo cual crea una centralidad en lo privado que aumenta la segregación estudiantil. Por otra parte, el Ministerio de Educación chileno, en el año 2017, presentó una reforma educativa, planteada para el 2020, la cual anunció que el Estado duplicará la inversión en las universidades de carácter público, de una manera transparente y sin ánimo de lucro.

Por otra parte, en México, fue expedida la Ley para la Coordinación de la Educación Superior (1978) y la Ley General de la Inclusión para las personas con discapacidad (2011); en estos documentos normativos, el Estado establece las bases para la distribución de la función educativa superior entre las diferentes federaciones y municipios, las cuales deben proteger y asegurar el pleno desarrollo de los derechos humanos y las libertades de las personas con discapacidad.

La síntesis contextual de los dos países latinoamericanos referenciados elucida un discurso sobre la inclusión, el cual enfatiza la urgencia de que las instituciones de educación superior se conviertan en garantes de derechos educativos de la población en condición de discapacidad. Como bien puede observarse, Chile y México, dentro de su marco legal, tienen planteamientos discursivos similares acerca de la educación inclusiva, que además coinciden con el discurso de la legislación colombiana.

Así las cosas, el mayor auge en materia de inclusión en Colombia, tiene sus asentamientos en la Ley 1618 del 2013, reglamentada por el Decreto 1421, que establece las disposiciones para garantizar el pleno ejercicio de los derechos de las personas con discapacidad y propone una serie de medidas y 
acciones afirmativas, las cuales permiten a las personas con discapacidad, bajo un marco de corresponsabilidad, ejercer sus derechos en igualdad de condiciones con las demás personas, en aspectos como el marco de la educación inclusiva y la atención educativa a la población con discapacidad.

El Decreto 1421 de 2017 se ha convertido en la orientación principal para concretar la norma en el contexto educativo colombiano, pues reglamenta el marco de la inclusión para toda la población con discapacidad.

No obstante, en el caso de Colombia, a diferencia de los dos países de referencia, la ruta global no está estipulada con claridad, pues existe otro tipo de población vulnerable que aún es marginada como colectivo. Por ejemplo, la consecuencia del proceso de paz que tuvo mayor impacto en el año 2012 generando el surgimiento de la población reinsertada, que fue acogida bajo el Decreto 128 de 2003, el explicita la política de reincorporación a la vida civil.

\section{Aspectos políticos, sociales y educativos que rigen la inclusión en la educación superior en Colombia, México y Chile}

La implementación de la inclusión en la educación superior en el marco político chileno tiene sus asentamientos legales en la Ley No. 20.422, la cual reguló todas las instituciones educativas del país; por otra parte, el programa de gobierno del presidente chileno Sebastián Piñera, en el año 2017, denominado "Construyamos tiempos mejores para Chile", plantea propuestas para la enseñanza del lenguaje de señas en las instituciones educativas e impulsa la implementación del plan nacional de accesibilidad en las ciudades, el cual establece el concepto de accesibilidad universal en espacios públicos; estos aspectos prometen mejorar los procesos de inclusión en la sociedad chilena.

En esta línea discursiva, Otondo (2018) y Castillo y Miranda (2018) manifiestan que las instituciones de educación superior chilenas establecen una serie de instrucciones para las personas con discapacidad que deseen entrar al proceso de selección universitaria. Desde estos planteamientos taxativos, los docentes y los administradores de la educación deben participar en procesos de actualización permanente para que su formación y su labor tengan como base funcional la inclusión educativa. La legislación chilena opta por demandar, de las facultades universitarias, propuestas alternativas que consoliden los procesos de inclusión de los estudiantes.

Dentro del aspecto social, autores como Vásquez y Alarcón (2016), Villafañe, Corrales y Soto (2016) y Henríquez (2011), declaran que las universidades en Chile buscan generar un discurso similar en el concepto de inclusión, por medio de la estructuración de un modelo interactivo del proceso como medio para la modernización del país. En esta figura comunicativa la discusión acerca de la inclusión, vincula al estudiante con discapacidad y al docente, 
mediante estrategias de formación. Pese a estas acciones, la carencia persiste en los vacíos que aún coexisten en la formación del profesorado universitario $\mathrm{y}$ en quienes administran y gestionan los procesos favorecedores de la permanecía y el tránsito por los diferentes niveles de las personas con discapacidad.

Siguiendo los parámetros mencionados, se revisó el contexto geográfico mexicano desde la esfera política que circunda los procesos de inclusión. En esta perspectiva, la Constitución Política mexicana, de 1917, prescribe que el Estado proveerá los apoyos requeridos para prevenir o compensar las desventajas o dificultades de las personas con discapacidad. De esta manera conseguirá su incorporación y participación plena en los ámbitos de la vida política, económica, social y cultural. El Estado también deberá elaborar y mantener actualizados, libros de texto gratuitos y demás materiales educativos, mediante procedimientos que permitan la participación de los diversos sectores sociales involucrados en la educación.

En este orden de ideas, desde la educación superior en México, la Universidad Veracruzana (2017) y la Universidad Autónoma del Estado de Morelos (2013) tienen, dentro de su PEI, políticas claras en relación con la inclusión de los estudiantes. Estas instituciones reciben apoyos académicos para realizar las actividades propias de cada estudiante; además, cuentan con un presupuesto para material educativo, infraestructura y tecnología, promulgando así el derecho a la educación. Igualmente, desarrollan estrategias de accesibilidad académica a los programas educativos, a través de sistemas alternativos o aumentativos de comunicación y la elaboración de informes anuales que evalúan el impacto de las políticas inclusivas del plantel educativo.

En una perspectiva social, la inclusión, en México, es tratada por autores como Cruz y Casillas (2017), Toscano et al. (2017) y García (2018), quienes exponen que este concepto ha tenido un avance importante en relación con las políticas inclusivas, aunque su desarrollo ha sido paulatino.

La mayoría de instituciones de educación superior no reconocen aún la existencia de los estudiantes con discapacidad, y muchas de ellas no están preparadas para cubrir las necesidades de esta población, siendo incapaces de ofrecerles una inclusión en todo el sentido educativo, que promueva la igualdad. Además, las propuestas que se plantean en el contexto de educación, están muy alejadas de las realidades sociales.

De otro lado, desde el punto de vista político, el proceso de inclusión en Colombia se fundamenta en las reformas educativas; el mayor auge de las políticas inclusivas está planteado en la Ley 1618 y su Decreto Reglamentario 1421 de 2017. Esta directiva legislativa amplió el panorama sobre el discurso de inclusión en la educación; en el caso de la educación superior, el mayor 
auge conceptual surgió en el año 2012 cuando se puso en entredicho la connotación de las necesidades educativas diversas (NED), para dar paso al concepto de barreras para el aprendizaje y a la participación como posibilidad de acceso, permanencia y promoción de los estudiantes, según sus particularidades.

El precepto de las políticas inclusivas, en el ámbito nacional, es parte articular del Plan Nacional de Desarrollo 2018-2022: Pacto por la equidad, que plantea como objetivo de la educación superior en el país: ofrecer oportunidades de acceso mediante el aumento de los recursos para la educación pública.

Asimismo, se articulan los planes de desarrollo municipal, garantizando una educación inclusiva desde la primera infancia hasta el final del ciclo educativo.

Finalmente, dentro del aspecto social, la inclusión educativa en Colombia, según Díaz y Rodríguez (2016) y Beltrán-Villamizar, Martínez-Fuentes y Vargas-Beltrán (2015), debe verse como una búsqueda de respuesta para atender a la diversidad de manera oportuna y eficaz; además, debe incluir procesos de socialización y proveer estrategias de sensibilización de toda la sociedad, para valorar las diferencias y evitar la estigmatización, como características fundantes de la vulnerabilidad social y educativa.

\section{Metodologia}

Para este trabajo se realizó un diagrama de los criterios de selección de los artículos el cual expone las razones por las cuales fueron escogidos o descartados. 
Figura 1. Metodología aplicada en el artículo.

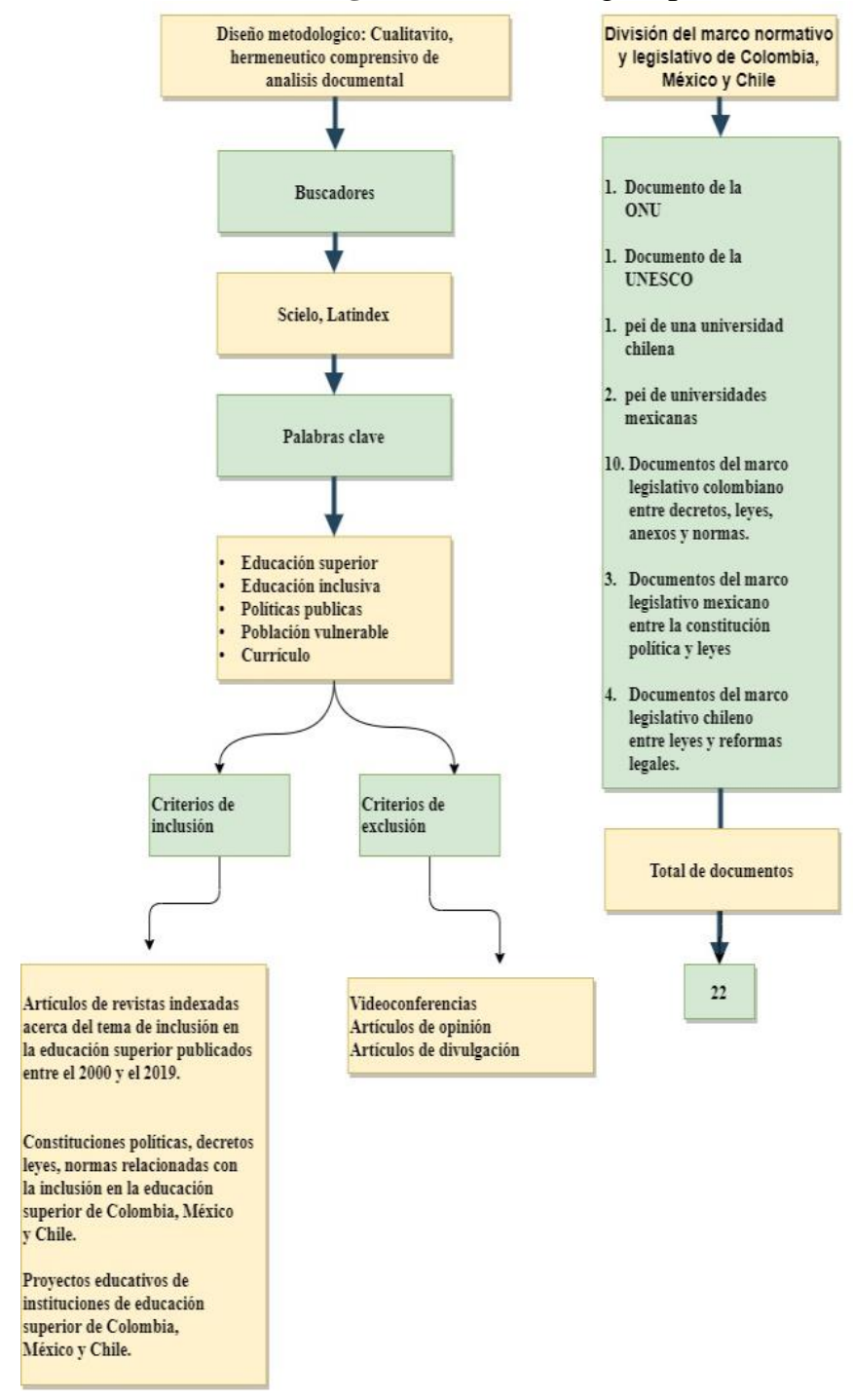

La primera búsqueda de información en las bases de datos seleccionadas tomó como referente las palabras clave arrojando un total, de 200 articulos.

y legislativo de Colombia,

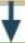

Después de una revisión inicial fueron descartados 31 articulos

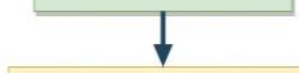

37. porque estaban enfocados en educación superior

29. porque no fueron realizados en los paises de interés.

31. porque estaban ubicados en bases de revistas de dirulgación.

23. porque no referenciaban el autor.

11. porque correspondian a opiniones de expertos

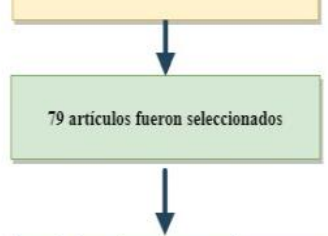

después de realizar una segunda Revisión se descartan 29 articulos Porque no tenian relacion con

- la inclusión en la educación superior

- los resultados de estas investigaciones no eran acordes al presente estudio

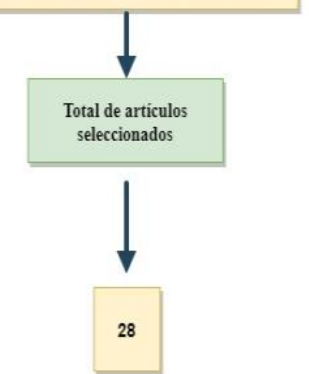

Fuente: Elaboración de los autores.

\section{Discusión}

El discurso de la inclusión desde los aspectos político, social y educativo se ha centralizado en la mejora de la calidad de vida de las personas con discapacidad, limitando su impacto a otras poblaciones. Autores como Echeita y Ainscow (2011) manifiestan que la inclusión es una acción de carácter global que busca disminuir las inequidades, discriminaciones y exclusiones, al fomentar el respeto por los derechos humanos, no solo para la población con discapacidad, dilucidando la importancia de incluir otro tipo de poblaciones vulnerables que requieren de adecuaciones especiales para su bienestar. 
En el marco legal de Chile y México se reconoce la educación inclusiva o integral solo a personas con discapacidad; en Colombia, por su parte, la educación inclusiva no solo contempla poblaciones en situación de discapacidad, como lo muestra la legislación. En relación con el avatar sociopolítico desatado por el conflicto-armado que ha desangrado el país, autores como Guerrero y Guerrero (2009) y Pachón, (2018), hacen énfasis en el aspecto educativo de la población reinsertada y desplazada, y manifiestan que la educación superior permite que estas personas se adapten a la vida social, por medio de métodos didácticos y procesos pedagógicos acordes con su realidad, permitiéndoles que tengan las mismas oportunidades que los demás ciudadanos y, de esta manera, contribuir a la reconstrucción del tejido social.

Las ideas que anteceden ponen de manifiesto que Colombia ha tenido un avance, no solamente discursivo, sino también pragmático, respecto a la inclusión educativa, a diferencia de Chile y México, pues se aprecia en el amplio abordaje de múltiples poblaciones en situación de vulnerabilidad, por medio de la educación y de su rol transformador. Escudero y Martínez (2011), basados en Raffo et al. (2009), manifiestan que la educación inclusiva no solo debe limitarse al derecho de las personas con capacidades diferentes a entrar en las escuelas; esta debe basarse en una reestructuración del sistema educativo el cual debe responder por todos los estudiantes.

En México, la inclusión en la educación, a partir de 1995, por medio del Programa Nacional para el bienestar y la incorporación al desarrollo de las personas con discapacidad, acoge los postulados de la Secretaría de Salud, el Programa Nacional para el Bienestar y la Incorporación al Desarrollo de las Personas con Discapacidad de la Presidencia de la República, y del Sistema Nacional de Desarrollo Integral para la Familia y la Secretaría de Educación Pública de México. Se diferencia de Colombia y Chile por ser el único país que posee educación inclusiva y educación especial, dándole a cada una roles, poblaciones y objetivos diferentes.

En Chile, el Gobierno manifiesta que se debe promover y respetar la diversidad de procesos y proyectos educativos institucionales, así como la diversidad cultural, religiosa y social de las familias que han elegido un proyecto diverso y determinado, y que son atendidas por él, en conformidad con la Constitución y las leyes; no obstante, Lissi et al. (2009) indican que solo el 6,6\% de los jóvenes con algún tipo de discapacidad accede a la universidad, y cuando lo hacen se encuentran con aspectos específicos que dificultan su inclusión. Las principales limitantes tienen que ver con la infraestructura, las barreras pedagógicas, los impedimentos comunicacionales y actitudinales; además, de este 6,6\% la tasa de egresados es incluso menor. 
Se puede determinar que, desde la educación superior, Colombia, México y Chile tienen aún grandes dificultades como garantes de derechos de la población en mención. Aunque aún no se puede hablar de universidades inclusivas, estos países continúan realizando acciones para garantizar, a las personas con capacidades diversas, el acceso y, ante todo, la permanencia y la promoción en los entornos educativos universitarios, atendiendo a sus características personales, sociales y económicas, y teniendo como meta el acceso a una vida laboral digna.

\section{Resultados}

Tomando como eje del análisis los tres países evocados, desde el año 2005 Colombia ha implementado, dentro de sus políticas inclusivas, poblaciones en situación de vulnerabilidad, como los desplazados por la violencia y los desmovilizados; por otra parte, México, en el 2004, impulsó estrategias y acciones básicas para orientar en las políticas inclusivas a las instituciones educativas de educación superior, pero solo en el 2013 se vieron resultados efectivos; Chile, por su parte, y a pesar de múltiples esfuerzos, solo en el 2015 empezó a implementar estrategias para la flexibilidad curricular, sustentado desde su legislación.

Ninguno de los tres países ha logrado implementar estrategias totalmente efectivas para el acceso a la educación superior para todo tipo de población, lo cual se ve reflejado en las siguientes acciones de cada país: México solo busca garantizar más cupos educativos, Chile cuenta con una de las cifras más bajas para el acceso a la educación superior de personas con algún tipo de discapacidad, y en Colombia solo el 3,4\% de esta población logra culminar sus estudios en educación superior.

\section{Conclusiones}

Teniendo en cuenta las diferentes concepciones desde el panorama educativo, el concepto de inclusión es visto como una discapacidad, limitando todas las poblaciones que están o deberían estar incluidas, por lo que se permite buscar alternativas que replanteen estrategias, las cuales visibilicen esta problemática desde una perspectiva más global.

La inclusión educativa no puede seguir limitándose conceptualmente a discapacidades de tipo físico, cognitivo, psicosocial y sensorial; debe ser percibida en todas sus dimensiones y tomada como excusa para generar posibilidades de crecimiento de las instituciones de educación superior, de tal forma que puedan consolidar unas acciones que eliminen contenidos memorísticos y se ocupen del desarrollo de procesos de pensamiento y de diseños universales, para que el aprendizaje no sea privilegio de unos pocos. De esta manera, los contextos universitarios se convertirán en semilleros multiculturales que ennoblecerán la sociedad diversa. 
Desde la implementación en la legislación de Colombia, Chile y México, en relación con una política inclusiva, se ha creado un banco de recursos educativos y económicos para el diseño de programas y planes inclusivos, en pro de fortalecer la calidad y equidad, aumentando así la accesibilidad a la educación superior de los estudiantes con discapacidad y en situación de vulnerabilidad.

La flexibilidad curricular, en pro de las adaptaciones a las realidades convergentes en Colombia, México y Chile, ha permitido la inclusión a la educación de las poblaciones con discapacidad y en situación de vulnerabilidad, que antes eran segregadas por el Estado y la sociedad, y que hoy en día, gracias a las políticas públicas inclusivas y la aceptación social, son garantes de sus derechos.

Colombia, México y Chile han hecho grandes esfuerzos para lograr que la educación inclusiva se articule significativamente a los retos que plantea a diario la educación superior, los cuales buscan mejorar el proceso de enseñanza-aprendizaje, la innovación y la practicidad, teniendo en cuenta las necesidades de los estudiantes, con el objetivo de visibilizar la diversidad que pueden encontrarse en un mismo escenario educativo.

\section{Referencias Bibliográficas}

Arizabaleta, S. L. y Ochoa, A. F. (2016). Hacia una educación superior inclusiva en Colombia. Pedagogía y Saberes, (45), 41-52.

Beltrán-Villamizar, Y. I., Martínez-Fuentes, Y. L. y Vargas-Beltrán, A. S. (2015). El sistema educativo Colombia no en el camino hacia la inclusión. Avances y retos. Educación y Educadores, 18(1), 62-75. Doi: https://doi.org/10.5294/edu.2015.18.1.4

Bocanegra, H. (2011). Reformas legales a la universidad en Colombia: Los síntomas de una crisis permanente y la cotidianidad de una política. Verba Iuruis, (25), 1139.

Brunner, J. J. (1990). Educación superior en América Latina cambios y desafíos. Chile: Fondo de Cultura Económica.

Carrizosa, J. (s.f.). La educación superior en el escenario del posconflicto: reflexión desde la Facultad de Medicina. Recuperado de https://bit.ly/38ml4tc

Castillo, P. y Miranda, C. (2018). Actitud hacia la inclusión de los estudiantes de pedagogía de una universidad estatal chilena. Revista Latinoamericana de Educación Inclusiva, 12(2), 133-148.

Ceballos, B. Y Acosta, N. (2015). Análisis documental sobre inclusión educativa en Colombia a partir de la producción académica de estudiantes de los programas de 
Licenciatura en Pedagogía Infantil y de Licenciatura en Educación Infantil de tres universidades (2009-2015). Revista Interacción, 14, 111-128.

Congreso de la República de Colombia. (28 de diciembre, 1992). Ley 30, por la cual se organiza el servicio público de la Educación Superior. Recuperado de http://www.secretariasenado.gov.co/senado/basedoc/ley_0030_1992.html

Congreso de la República de Colombia. (8 de febrero, 1994). Ley 115, por la cual se expide la ley general de educación. Recuperado de http://www.secretariasenado.gov.co/senado/basedoc/ley_0115_1994.html

Congreso de la República de Colombia. (11 de octubre 1996). Ley 324, por la cual se crean algunas normas a favor de la población sorda. Recuperado de http://www.secretariasenado.gov.co/senado/basedoc/ley_0324_1996.html

Congreso de la República de Colombia. (7 de febrero 1997). Ley 361, Por la cual se establecen mecanismos de integración social de las personas en situación de discapacidad y se dictan otras disposiciones. Recuperado de http://www.secretariasenado.gov.co/senado/basedoc/ley 0361 1997.html

Congreso de la República de Colombia. (27 de febrero, 2013). Ley Estatutaria 1618, por medio de la cual se establecen las disposiciones para garantizar el pleno ejercicio de los derechos de las personas con discapacidad. Recuperado de http://www.secretariasenado.gov.co/senado/basedoc/ley_1618_2013.html

Congreso de los Estados Unidos mexicanos. (29 de diciembre, 1978). Ley para la coordinación de la educación superior. Recuperado de http://www.diputados.gob.mx/LeyesBiblio/pdf/182.pdf

Congreso General de los Estados Unidos Mexicanos (30 de mayo, 2011). Ley general para la inclusión de las personas con discapacidad. Recuperado de http://www.diputados.gob.mx/LeyesBiblio/pdf/LGIPD_120718.pdf

Congreso Nacional de Chile. (28 de julio, 2009). Ley 20.370, Ley General de Educación. Recuperado de http://www.leychile.cl/Navegar?idNorma=1006043\&idParte

Congreso Nacional de Chile. (21 de enero, 2010). Ley 20.422, establece normas sobre igualdad de oportunidades e inclusión social de personas con discapacidad. Recuperado de https://www.leychile.cl/Navegar?idNorma=1010903\&idParte

Consejería Presidencial para el Desarrollo Institucional. (1996). Colombia: al filo de la oportunidad. Bogotá, Colombia: Tercer Mundo Editores.

Constitución Política de Colombia. (1991). Gaceta Constitucional No. 116 de 20 de julio de 1991.

Cruz, R. y Casillas, M. Á. (2017). Las instituciones de educación superior y los estudiantes con discapacidad en México. Revista de La Educación Superior, 46(181), 37-53. Doi: https://doi.org/10.1016/j.resu.2016.11.002 
Díaz, L. E. y Rodríguez, L. P. (2016). Educación inclusiva y diversidad funcional: Conociendo realidades, transformando paradigmas y aportando elementos para la práctica. Zona Próxima, (24), 43-60.

Echeita, G. y Ainscow, M. (2011). La educación inclusiva como derecho. Marco de referencia y pautas de acción para el desarrollo de una revolución pendiente. Tejuelo, (12), 26-46.

Escudero, J. M. y Martínez, B. (2011). Educación inclusiva y cambio escolar. Revista Iberoamericana de Educación, (55), 85-105.

Flórez, J. R. (2016). La política pública de educación en Colombia: gestión del personal docente y reformas educativas globales en el caso Colombia No. Academia \& Derecho, (13), 309-332. Doi: https://doi.org/10.18041/22158944/academia.13.313

Forero, R., González, L. y Gómez, V. M. (2007). Formación de élites y educación superior: meritocracia y reclutamiento en el Departamento Nacional de Planeación. Revista Colombiana de Sociología, (28), 161-180.

García, I. (2018). La educación inclusiva en la Reforma Educativa de México. Revista Nacional e Internacional de Educación Inclusiva, 11(2), 49-62.

García, I., Escalante, I., Escandón, M. C., Fernández, L. F., Mustri, A. y Toulet, I. (2003). Proyecto de investigación integración educativa. Perspectiva internacional y nacional. Informe final de investigación (Ciclos escolares 19951996).

Gobierno de Chile. (2017). Programa de Gobierno 2018-2022. Construyamos tiempos mejores para Chile. Recuperado de https://planipolis.iiep.unesco.org/sites/planipolis/files/ressources/chileprogramad egobiernodesebastianpinera.pdf

Gómez, V. M. (2000). Cuatro temas críticos de la educación superior en Colombia: Estado, instituciones, pertinencia y equidad social. Colombia: Universidad Nacional de Colombia, ASCUN y Alfaomega.

Guerrero, M. E. y Guerrero, M. H. (2009). Acceso a la educación de la población desplazada por el conflicto armado en Colombia 1999- 2009. Studiositas, 4(2), 67-76.

Henríquez, C. P. (2011). Educación en Chile: ¿ Inclusión o exclusión ? Tejuelo, 12, 47-79.

Lissi, R., Zuzulich, S., Salinas, M., Achiardi, C., Hojas, A. M. y Pedrals, N. (2009). Discapacidad en contextos universitarios: experiencia del Piane UC. Calidad en la Educación, 307(30), 305-324. Doi: http://dx.doi.org/10.31619/caledu.n30.183

Marcel, M. y Tokman, C. (2005). ¿Cómo se financia la educación en Chile? Chile: Ministerio de Hacienda. 
Ministerio de Educación de Chile. (2017). Reforma educacional: ley de inclusión escolar. Santiago de Chile, Chile. Recuperado de https://www.mineduc.cl/wpcontent/uploads/sites/19/2018/03/libro_Inclusio\%CC $\% 81$ _final.pdf

Mollis, M. (2006). Geopolítica del saber: biografías recientes de las universidades latinoamericanas. En Clacso (Ed.), Universidad e Investigación Científica (pp. 85101). Buenos Aires, Argentina: Clacso.

Organización de las Naciones Unidas -ONU-. (2018). Estrategia de las Naciones Unidas para la inclusión de la discapacidad. Recuperado de https://www.un.org/en/content/disabilitystrategy/assets/documentation/UN_Disa bility_Inclusion_Strategy spanish.pdf

Organización de las Naciones Unidas para la Educación, la Ciencia y la Cultura UNESCO-. (1960). Inclusión en la Educación. Recuperado de https://es.unesco.org/themes/inclusion-educacion

Otondo, M. (2018). Inclusión de estudiantes con discapacidad en educación superior. Espacios, $39(49)$ Recuperado de http://www.revistaespacios.com/a18v39n49/a18v39n49p06.pdf

Pachón, W. (2018). Inclusión social de actores del conflicto armado colombiano: Retos para la educación superior. Desafíos, 30(1), 279-308.

Presidencia de la República de Colombia. (22 de enero, 2003). Decreto 128, por el cual se reglamenta la Ley 418 de 1997, prorrogada y modificada por la Ley 548 de 1999 y la Ley 782 de 2002 en materia de reincorporación a la sociedad civil. Recuperado http://wp.presidencia.gov.co/sitios/normativa/leyes/Documents/Juridica/DECRE TO\%20128\%20de\%202003.pdf

Presidencia de la República de Colombia. (29 de agosto, 2017). Decreto 1421, por el cual se reglamenta en el marco de la educación inclusiva la atención educativa a la población con discapacidad. Recuperado de http://es.presidencia.gov.co/normativa/normativa/DECRETO\%201421\%20DEL \%2029\%20DE\%20AGOSTO\%20DE\%202017.pdf

Presidencia de la República de Colombia. (2018). Plan Nacional de Desarrollo 2018-2022: Pacto por la equidad. Bogotá, Colombia: Departamento Nacional de Planeación.

Programa de las Naciones Unidas para el Desarrollo -PNUD- (s.f.). Objetivos de Desarrollo Sostenible. Recuperado de https://www.undp.org/content/undp/es/home/sustainable-developmentgoals.html

Queupil, J. P. y Durán, F. (2018). El principio de inclusión: Similitudes y diferencias en la educación escolar y superior en Chile. Revista Latinoamericana de Educación Inclusiva, 12(1), 111-128. 
Rivas, A. y Sánchez, B. (2016). Políticas y resultados educativos en América Latina: un mapa comparado de siete países (2000-2015). RELIEVE, 22(1), 1-30.

Toscano, B. A., Ponce, J. C., Cruz, A. de loS Á., Zapién, A., Contreras, G. y Pérez, J. C. (2017). Análisis de la inclusión en la educación superior en México. Una propuesta de indicadores para los organismos acreditadores. Tecnología Educativa Revista CONAIC, 4(2), 35-51.

Universidad Autónoma del Estado de Morelos. (2013). Programa universitario para la inclusión educativa de personas con discapacidad. Recuperado de https://www.uaem.mx/vida-universitaria/unidad-para-la-inclusion-educativa-yatencion-a-la-diversidad/

Universidad Veracruzana. (2017). Programa universitario de educación inclusiva (2016-2017).

Recuperado https://www.uv.mx/cendhiu/files/2016/10/Programa-Universitario-de-

Educacion-Inclusiva-.pdf

Vásquez, B. y Alarcón, E. (2016). La inclusión en las universidades chilenas: del discurso a las interacciones prácticas, políticas y culturales. Pensamiento Educativo, 53(2), 1-18. Doi: https://doi.org/10.7764/pel.53.2.2016.9

Villafañe, G. P., Corrales, A. A. y Soto, V. J. (2016). Estudiantes con discapacidad en una universidad chilena: Desafíos de la inclusión. Revista Complutense de Educación, 27(1), 353-372.

Villalobos, C. y Quaresma, M. L. (2015). Sistema escolar chileno: Características y consecuencias de un modelo orientado al mercado. Convergencia, 22(69), 63-84. 